\title{
Measurements of Viscoelasticity by the Vibrating-Reed Method at Very Low Frequencies
}

\author{
Shigeharu ONOGI, Takeshi Kondo*, and Yoshishige TABATA \\ Department of Polymer Chemistry, Kyoto University, Kyoto, Japan.
}

(Received March 24, 1972)

\begin{abstract}
Measurements of the elasticity or viscoelasticity of various materials by the vibrating-reed method at very low frequencies require the use of long and heavy samples. The effect of gravity on these samples has been considered theoretically, and new equations for the storage and loss moduli have been derived. Vibrating-reed measurements were carried out with several kinds of materials at frequencies ranging from about 1 to $20 \mathrm{~Hz}$. The experimental results indicate that the above equations are satisfactory in practice.
\end{abstract}

KEY WORDS Viscoelasticity / Elasticity / Modulus of Elasticity / Vibrating-Reed Method / Bending Modulus / Polymer Films / Fabrics /

The vibrating-reed method is one of the simplest methods for measuring the elasticity or viscoelasticity of solid materials; it has been applied to various kinds of materials including fibers, films, papers, and fabrics ${ }^{1-13}$. This method is usually used in the audiofrequency range, but in many cases measurements at very low frequencies are desired. In making such measurements very long, and hence very heavy sample reeds must be employed to obtain the resonance frequencies or resonance curves from which viscoelastic functions, such as the storage and loss moduli, dynamic viscosity, and mechanical loss tangent can be evaluated.

One of the very important assumptions upon which the vibrating-reed method is based is that the mass of the reed is negligibly small as compared with that of the vibrator and that hence the effect of gravity can be ignored. However, the use of long and heavy reeds at very low frequencies tends to conflict with this assumption. In such cases, the effect of gravity should be taken into considration.

The present paper is intended to offer some experimental evidence for the existence of the effect of gravity and to present a new theory of the vibrating-reed method when it is employed at very low frequencies in which the effect of gravity is considered.

* Present address: Central Research Laboratories, Unitika Ltd., Uji, Kyoto, Japan.

\section{THEORETICAL}

The transverse vibration of a viscoelastic beam or reed having a transverse dimension which is small in comparison with both its length and the wave legth can be expressed by

$$
\rho \frac{\partial^{2} y}{\partial t^{2}}+E_{\kappa}^{2} \frac{\partial^{4} y}{\partial x^{4}}+\eta^{\prime} \kappa^{2} \frac{\partial^{5} y}{\partial x^{4} \partial t}=0
$$

where $x$ is the axis of the beam in the position of equilibrium and $y$ the axis perpendicular to it and parallel to the plane of symmetry. ${ }^{14,15}$ Here $t$ is time, $\rho$ density, $E^{\prime}$ modulus of elasticity (bending modulus), $\eta^{\prime}$ coefficient of viscosity, and $\kappa^{2}$ moment of inertia of the cross section around the neutral axis per cross-sectional area. This equation was solved by Horio and Onogi $i^{16}$ for the case of forced vibration to give

$$
E^{\prime}=\frac{4 \pi^{2} \rho l^{4}}{a_{0}^{4} \kappa^{2}}\left[\nu_{\mathrm{r}}{ }^{2}+\frac{1}{8}(\Delta \nu)^{2}\right]
$$

and

$$
E^{\prime \prime}=\frac{4 \pi^{2} \rho l^{4}}{a_{0}^{4} \kappa^{2}} \nu_{\mathrm{r}} \Delta \nu
$$

or

$$
\eta^{\prime}=\frac{2 \pi \rho l^{4}}{a_{0}{ }^{4} \kappa^{2}} \Delta \nu
$$

where $E^{\prime \prime}$ is the loss modulus or the imaginary part of the complex modulus, $\nu_{r}$ is the resonance frequency, $\Delta \nu$ is the band width, $l$ is the effective length, and 


\section{S. Onogi, T. Kondo, and Y. TABATA}

$$
a_{0}=1.875,4.694,7.855, \ldots
$$

When such a viscoelastic beam is held vertically and affected by gravity, eq 1 should contain another term for the effect of gravity; in that case it becomes too complex to be solved even in an approximate manner. Therefore, we consider an elastic beam affected by gravity. The equation of motion for the beam is given by

$$
E^{\prime} \kappa^{2} \frac{\partial^{4} y}{\partial x^{4}}-\frac{\partial}{\partial x}\left(T_{\frac{\partial y}{\partial x}}\right)+\rho \frac{\partial^{2} y}{\partial t^{2}}=0
$$

where

$$
T=\rho g(l-x)
$$

and $g$ is the acceleration of gravity.

Assuming that $y$ is presented by $Y e^{i \omega t}$ in the stationary state of motion, $i$ being $(-1)^{1 / 2}$, we obtain

$$
\begin{gathered}
\frac{\mathrm{d}^{4} Y}{\mathrm{~d} x^{4}}-\frac{\rho g}{E^{\prime} \kappa^{2}}(l-x) \frac{\mathrm{d}^{2} Y}{\mathrm{~d} x^{2}}+\frac{\rho g}{E^{\prime} \kappa^{2}} \frac{\mathrm{d} Y}{\mathrm{~d} x} \\
-\frac{\rho \omega^{2}}{E^{\prime} \kappa^{2}} Y=0
\end{gathered}
$$

This equation cannot be solved rigorously. Therefore, we employ the following approximation:

$$
\frac{\mathrm{d}^{4} Y}{\mathrm{~d} x^{4}}=\frac{a_{0}^{4}}{l^{4}} Y
$$

which is the case where no effect of gravity exists. Then, eq 8 becomes

$$
g(l-x) \frac{\mathrm{d}^{2} Y}{\mathrm{~d} x^{2}}-g \frac{\mathrm{d} Y}{\mathrm{~d} x}+\left(\omega^{2}-\frac{a_{0}{ }^{4} E^{\prime} \kappa^{2}}{\rho l^{4}}\right) Y=0
$$

When we put

and

$$
z=l-x,
$$

$$
u=2\left(\frac{z}{g}\right)^{1 / 2}\left(\omega^{2}-\frac{a_{0}^{4} E^{\prime} \kappa^{2}}{\rho l^{4}}\right)^{1 / 2}
$$

eq 10 becomes

$$
\frac{\mathrm{d}^{2} Y}{\mathrm{~d} u^{2}}+\frac{1}{u} \frac{\mathrm{d} Y}{\mathrm{~d} u}+Y=0
$$

This equation is a Bessel's differential equation and its solution can be given by a Bessel function

$$
Y=\sum_{m=0}^{\infty} \frac{(-1)^{m}}{m !^{2}}\left(\frac{l}{g}\right)^{m}\left(\omega^{2}-\frac{a_{0}^{4} E^{\prime} \kappa^{2}}{\rho l^{4}}\right)^{m}=0
$$

The numerical calculation of this function gives

$$
\frac{l}{g}\left(\omega^{2}-\frac{a_{0}^{4} E^{\prime} \kappa^{2}}{\rho l^{4}}\right) \cong 1.45
$$

And hence we obtain the following equation for the resonance frequency $\nu_{r}$

$$
\nu_{\mathrm{r}}^{2} l \cong E^{\prime} \frac{a_{0}^{4} \kappa^{2}}{4 \pi^{2} \rho l^{3}}+36\left(\mathrm{~cm} / \mathrm{sec}^{2}\right)
$$

or

$$
E^{\prime} \cong \frac{4 \pi^{2} \rho l^{3}}{a_{0}{ }^{4} \kappa^{2}}\left(\nu_{\mathrm{r}}^{2} l-36\right)
$$

Equation 12 is valid only when $l$ is large. When $l$ is smaller, we obtain

$$
E^{\prime} \cong \frac{4 \pi^{2} \rho l^{3}}{a_{0}{ }^{4} \kappa^{2}}\left(\nu_{\mathrm{r}}{ }^{2} l-50\right)
$$

\section{EXPERIMENTAL}

By means of a vibrating-reed apparatus, re-

Table I. The thickness $d$ and density $\rho$ of aluminium foils, Al-1 and Al-2, and nichrome wire, $\mathrm{Ni}-\mathrm{Cr}$

\begin{tabular}{ccc}
\hline Sample & $d \times 10^{2}, \mathrm{~cm}$ & $\rho, \mathrm{g} / \mathrm{cm}^{3}$ \\
\hline Al-1 & 0.215 & 2.69 \\
Al-2 & 0.976 & 2.69 \\
Ni-Cr & $1.73^{\mathrm{a}}$ & 8.40 \\
\hline
\end{tabular}

a $d$, radius.

Table II. The thickness $d$ and density $\rho$ of poly(vinyl chloride) (PVC) and polyethylene (PE-1 and PE-2) films

\begin{tabular}{ccc}
\hline Sample & $d \times 10^{2}, \mathrm{~cm}$ & $\rho, \mathrm{g} / \mathrm{cm}^{3}$ \\
\hline PVC & 1.96 & 1.26 \\
PE-1 & 0.526 & 0.899 \\
PE-2 & 0.740 & 0.915
\end{tabular}

Table III. The thickness $d$ and apparent density $\rho$ of fabric samples

\begin{tabular}{cccc}
\hline Sample $^{a}$ & $\begin{array}{c}\text { Blending ratio, } \\
\text { Acrylic wt } \%\end{array}$ & $\begin{array}{c}d \times 10^{2}, \\
\mathrm{~cm}\end{array}$ & $\begin{array}{c}\rho, \\
\mathrm{g} / \mathrm{cm}^{3}\end{array}$ \\
\hline $\mathrm{S}_{1}$ & 0 & 3.95 & 0.621 \\
$\mathrm{~S}_{2}$ & 25 & 3.85 & 0.567 \\
$\mathrm{~S}_{3}$ & 50 & 3.91 & 0.555 \\
$\mathrm{~S}_{4}$ & 75 & 3.93 & 0.541 \\
$\mathrm{~S}_{5}$ & 100 & 4.04 & 0.520 \\
\hline $\mathrm{A}_{1}$ & 0 & 2.95 & 0.575 \\
$\mathrm{~A}_{2}$ & 50 & 3.50 & 0.515 \\
$\mathrm{~A}_{3}$ & 100 & 3.57 & 0.498 \\
\hline
\end{tabular}

a The $\mathrm{S}$ series are blends of wool and acrylic fibers, and the A series are blends of polyester and acrylic fibers. 
sonance curves for various materials were measured in a frequency range between about 1.0 and $20 \mathrm{~Hz}$. The measuring temperature and humidity were kept constant at $25^{\circ} \mathrm{C}$ and $65 \% \mathrm{RH}$.

In Tables I to III are listed the thicknesses and densities of the samples used in this study.

\section{RESULTS AND DISCUSSION}

When the band width in eq 2 is negligibly small and the modulus $E^{\prime}$ is constant independent of frequency in the frequency range covered, the resonance frequency $\nu_{\mathrm{r}}$ plotted against $1 / l^{2}$ should give a straight line passing

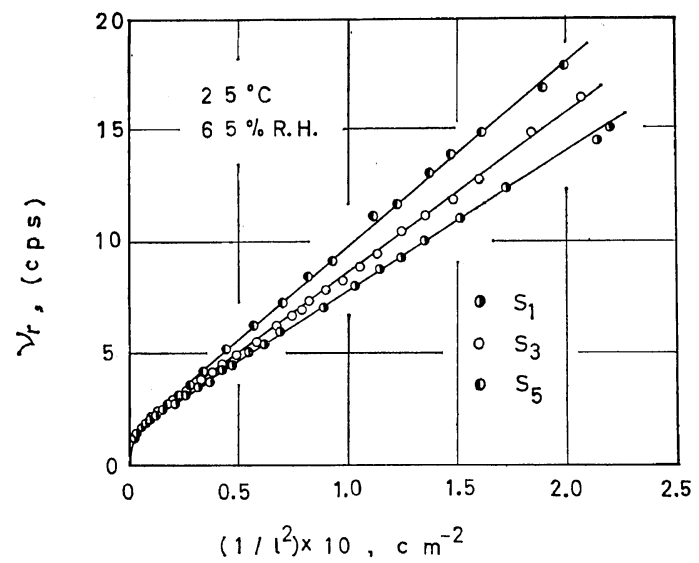

Figure 1. $\nu_{\mathrm{r}} v s .1 / l^{2}$ for the fabric samples $\mathrm{S}_{1}, \mathrm{~S}_{3}$, and $\mathrm{S}_{5}$.

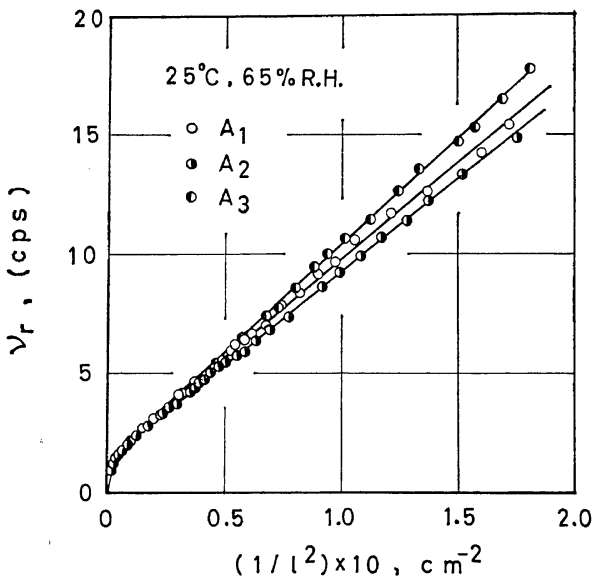

Figure 2. $\nu_{r}$ vs. $1 / /^{2}$ for the fabric samples $\mathrm{A}_{1}, \mathrm{~A}_{2}$, and $\mathbf{A}_{3}$. through the origin. This type of plot is usually made in order to determine the modulus from slope $m$ of the straight line by the following equation $^{11}$ :

$$
E^{\prime}=\frac{m^{2} \rho}{(0.162 d)^{2}} \quad \begin{aligned}
& \text { for the fundamental oscil- } \\
& \text { lation, where } a_{0}=1.875
\end{aligned}
$$

where $d$ is the thickness of a beam having rectangular cross section.

In the usual measurements in the audiofrequency range, $\nu_{\mathrm{r}}$ vs. $1 / l^{2}$ curves for various

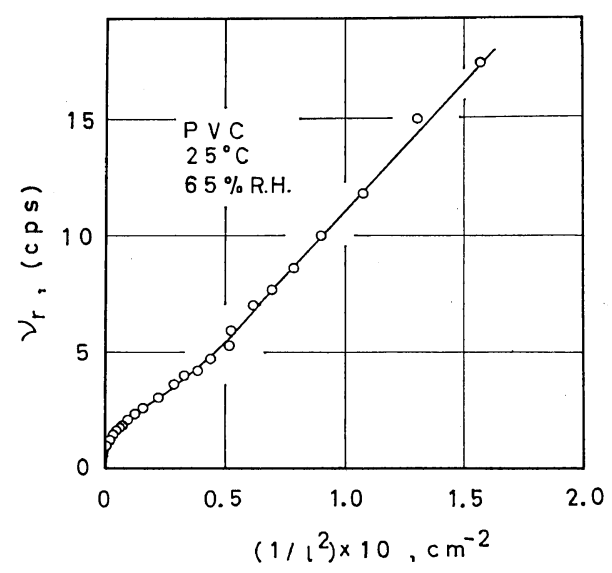

Figure 3. $\nu_{\mathrm{r}}$ vs. $1 / l^{2}$ for the poly(vinyl chloride) film.

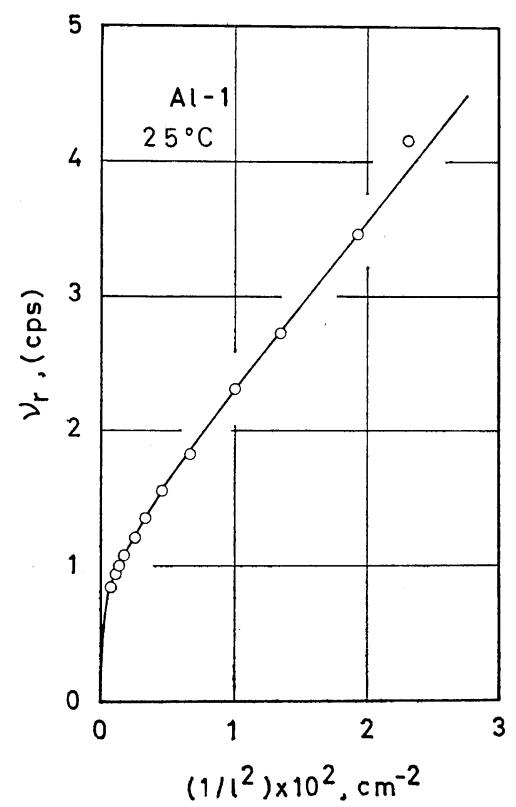

Figure 4. $\nu_{\mathrm{r}} v s .1 / l^{2}$ for the aluminium foil Al-1. 


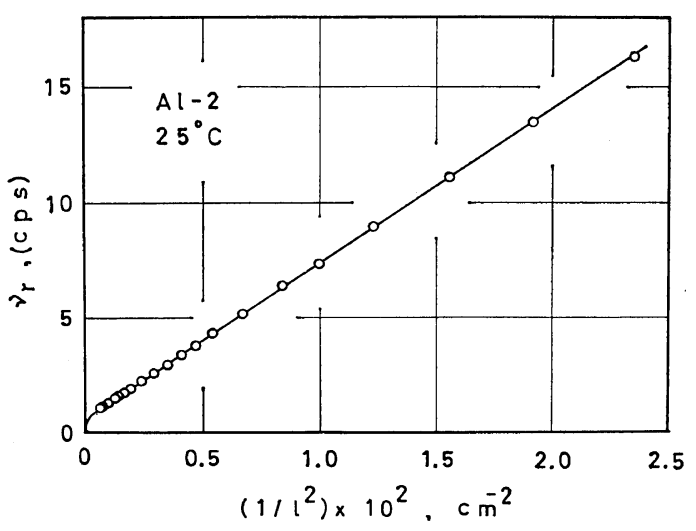

Figure 5. $\nu_{\mathrm{r}}$ vs. $1 / l^{2}$ for the aluminium foil $\mathrm{Al}-2$.

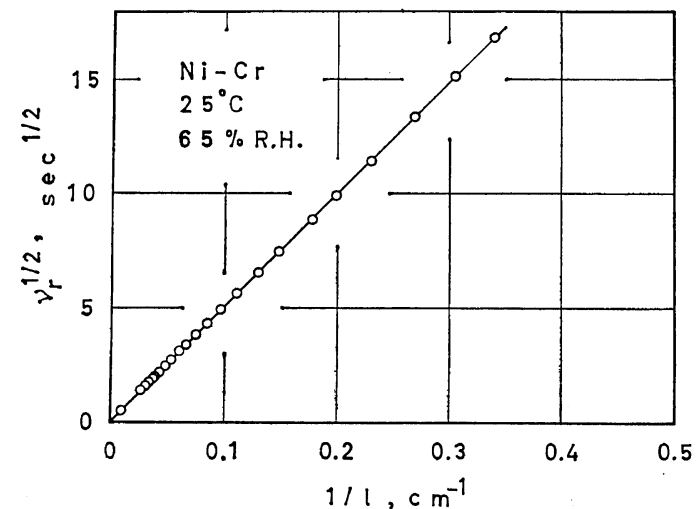

Figure 6. $\nu_{r}^{1 / 2}$ vs. $1 / l$ for the nichrome wire.

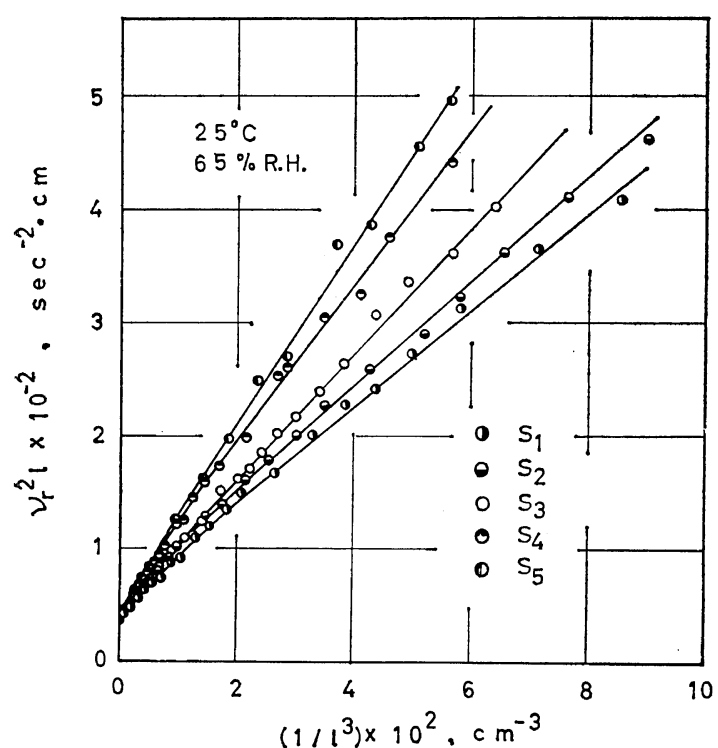

Figure 7. $\nu_{\mathrm{r}}^{2} l$ vs. $1 / l^{3}$ for the fabric samples $\mathrm{S}_{1}, \mathrm{~S}_{2}$, $\mathrm{S}_{3}, \mathrm{~S}_{4}$, and $\mathrm{S}_{5}$. materials including fibers, papers, fabrics, films, and sheets are linear. But eq 2 is no longer valid at frequencies as low as 1 to $20 \mathrm{~Hz}$, and $\nu_{r}$ plotted against $1 / l^{2}$ cannot give a straight line passing through the origin, as is shown in Figures 1 to 5 for the various kinds of materials

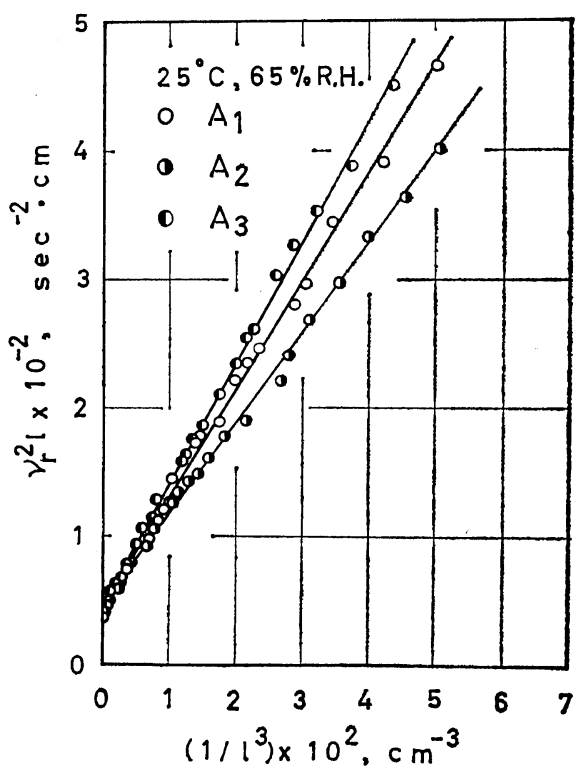

Figure 8. $\nu_{\mathrm{r}}^{2} l v s .1 / l^{3}$ for the fabric samples $\mathbf{A}_{1}, \mathbf{A}_{2}$, and $\mathrm{A}_{3}$.

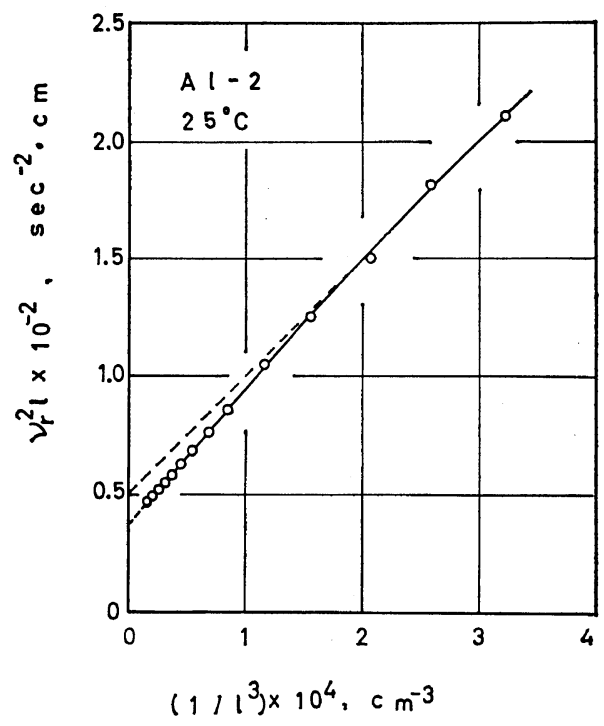

Figure 9. $\nu_{\mathrm{r}}^{2} l$ vs. $1 / l^{3}$ for the aluminum foil Al-2.

Polymer J., Vol. 4, No. 1, 1973 
Measurements of Viscoelasticity by the Vibrating-Reed Method at Very Low Frequencies

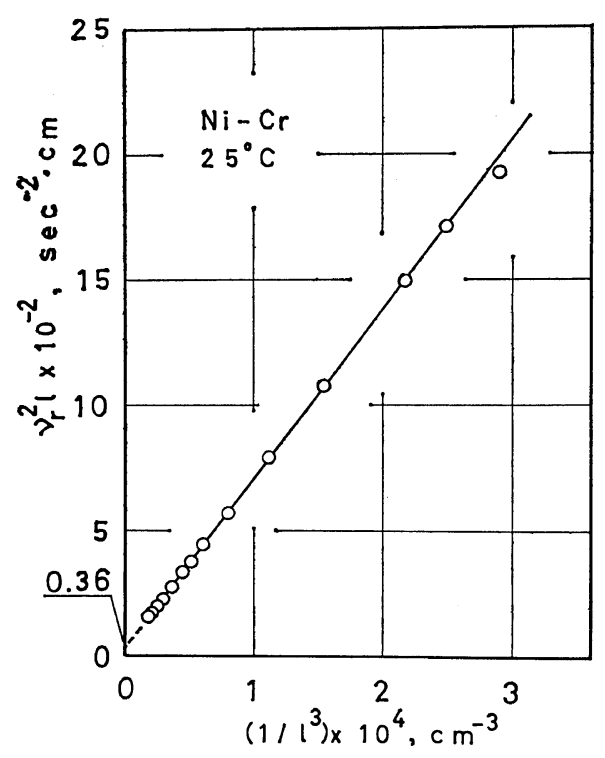

Figure 10. $\nu_{\mathrm{r}}^{2} l v s .1 / l^{3}$ for the nichrome wire.

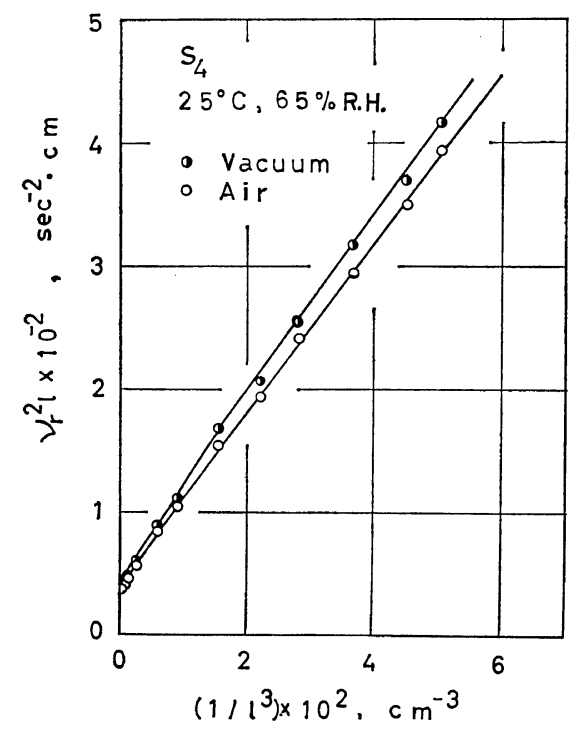

Figure 11. $\nu_{\mathrm{r}}^{2} l v s .1 / 3$ for the fabric sample $\mathrm{S}_{4}$.

mentioned above. The $\nu_{\mathrm{r}}^{1 / 2}$ vs. $1 / l$ curve for the nichrome wire shown in Figure 6 seems to be a straight line passing through the origin. However, this is only apparent, as is noted below.

On the other hand when eq 13, including the effect of gravity, is valid $\nu_{\mathrm{r}}^{2} l$ plotted against $1 / l^{3}$ should give a straight line having the con-

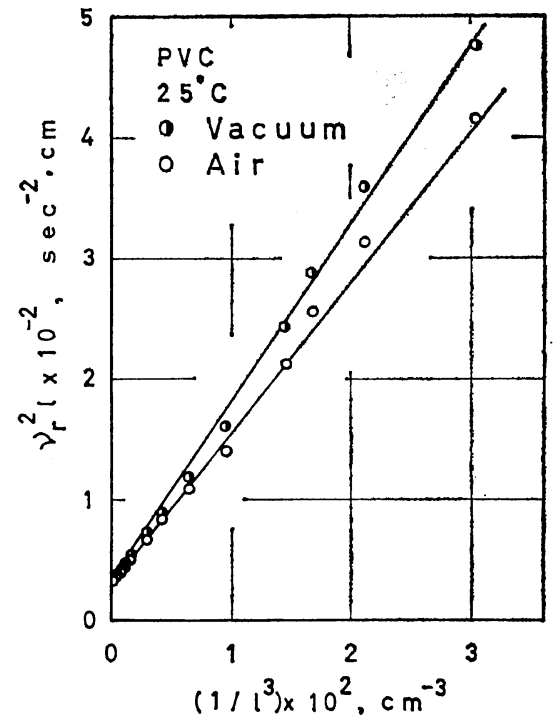

Figure 12. $\nu_{\mathrm{r}}^{2} l$ vs. $1 / l^{3}$ for the poly(vinyl chloride) film.

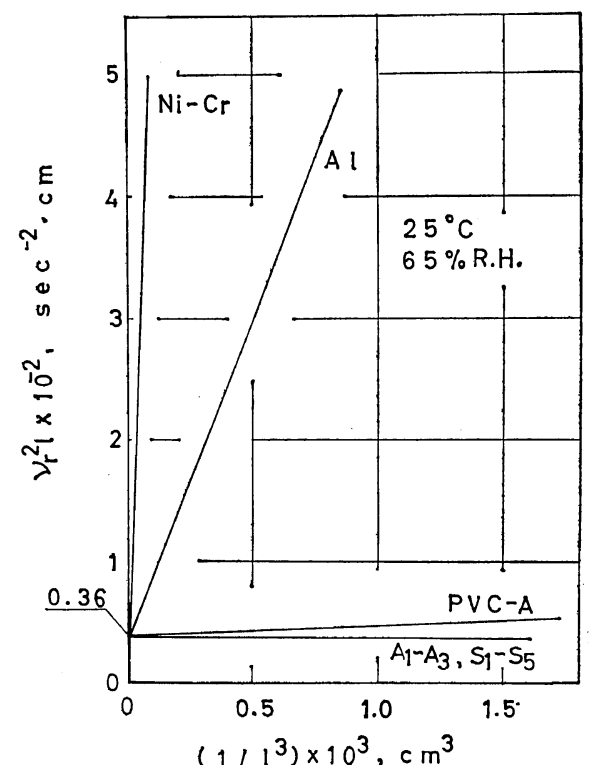

Figure 13. $\nu_{\mathrm{r}}^{2} l$ vs. $1 / l^{3}$ for various samples.

stant intercept of 36 independent of the nature of the material. Figure 7 shows an example of such a plot for the $S$ series of fabrics. As is evident from this figure, the plot is almost linear but not completely, so, indicating that eq 13 holds only approximately. Nevertheless, the plot has the intercept of 36 as is required 
by eq 13. Furthermore, the extraporation of a linear portion of the plot at higher $1 / l^{3}$ or smaller $l$ values gives the intercept of about 50 , which shows a good agreement with the 50 in eq 14.

Similar results for the other samples are shown in Figures 8 to 12. Some results in these figures were obtained by experiments in vacuo which gave resonance frequencies somewhat higher than those obtained in air, because of the absence of the frictional resistance of air. Furthermore, one notices that some curves in these figures are almost linear. It is noteworthy that the intercepts of all the curves including that for the nichrome wire mentioned above are 36, which is consistent with eq 13 . This is clearer in Figure 13, where the $\nu_{\mathrm{r}}^{2} l v s$. $1 / l^{3}$ curves for five different materials are plotted together. It is also evident from this figure that the slope of the curves is different from material to material, because the moduli of elasticity for these materials are quite different. From the slopes $m^{\prime}$ of these curves, the moduli $E^{\prime}$ can be evaluated using

$$
E^{\prime}=\frac{4 \pi^{2} \rho}{a_{0}{ }^{4} \kappa^{2}} m^{\prime}
$$

An example of $E^{\prime}$ thus determined for the sample $\mathrm{S}_{3}$ is shown by the open circle in Figure 14. As is clear from this figure, $E^{\prime}$ is almost constant independent of the sample length $l$ or the measuring frequency. But $E^{\prime}$ evaluated from

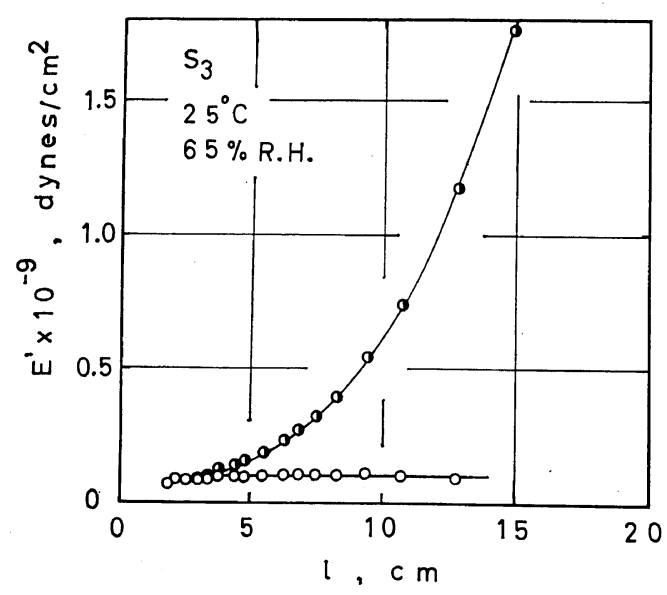

Figure 14. $E^{\prime}$ of $S_{3}$ determined from eq 2 (half closed circles) and eq 13 (open circles).

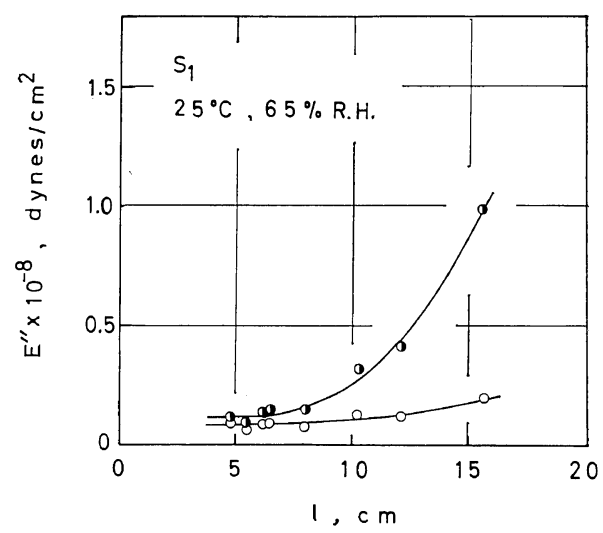

Figure 15. $E^{\prime \prime}$ of $S_{1}$ determined from eq 3 (half closed circles) and eq 17 (open circles).

the slope $m$ of the $\nu_{\mathrm{r}} v s .1 / l^{2}$ plot using eq 15 increases very rapidly with increasing sample length, as is shown by half closed circles in the same figure. The modulus determined by eq 13 seems to be more reasonable, because the rapid increase of the modulus in a narraw frequency range is impossible.

Next, the determination of the loss modulus $E^{\prime \prime}$ may be discussed. When we employ eq 3 to determine $E^{\prime \prime}$, the evaluated value of $E^{\prime \prime}$ increases very unreasonably with increasing sample length, as can be seen from the typical example for the sample $S_{1}$ shown in Figure 15. This indicates that the effect of the gravity should be considered here again.

The only difference between eq 2 and eq 13 for $E^{\prime}$ was that $\nu_{\mathrm{r}}^{2}$ in eq 2 was replaced with $\left(\nu_{\mathrm{r}}^{2}-36 / l\right)$ in eq 13 , neglecting $\Delta \nu$ in eq 2 . Therefore, when we replace $\nu_{r}$ in eq 3 with $\left(\nu_{r}{ }^{2}-\right.$ $36 / l)^{1 / 2}$, we obtain

$$
E^{\prime \prime}=\frac{4 \pi^{2} \rho l^{4}}{a_{0}^{4} \kappa^{2}}\left(\nu_{\mathrm{r}}{ }^{2}-36 / l\right)^{1 / 2} \Delta \nu
$$

Using this equation, $E^{\prime \prime}$ for the sample $S_{1}$ was evaluated and compared with that from eq 3 in Figure 15. As is evident from this figure, $E^{\prime \prime}$ from eq 17 increases only slightly with increasing $l$ or decreasing frequency, indicating that eq 17 is more reasonable than eq 3 .

Summarizing all these results, it can be concluded that eq 13 and 17 rather than eq 2 and 3 should be employed to evaluated $E^{\prime}$ and $E^{\prime \prime}$ from $\nu_{\mathrm{r}}$ and $\Delta_{\nu}$ measured at very low frequencies, 
Measurements of Viscoelasticity by the Vibrating-Reed Method at Very Low Frequencies

though eq 13 and 17 are not completely rigorous.

Acknowledgement. This study was supported by a grant from the Japan Research Association for Textile End-Uses and the Japan Chemical Fibres Association, for which the authors are grateful.

\section{REFERENCES}

1. J.P.A. Lochner, J. Text. Inst., 40, T220 (1949).

2. J. W. Ballou and J. C. Smith, J. Appl. Phys., 20, 493 (1949).

3. K. W. Hillier, Proc. Phys. Soc. (London), B64 (1951).

4. M. Horio, S. Onogi, C. Nakayama, and K. Yamamoto, J. Appl. Phys., 22, 966 (1951).

5. S. Onogi, S. Ando, and I. Suginaka, Sen-i Gakkaishi, 9, 617 (1953); 10, 32 (1954); 10, 390 (1954).
6. E. M. Kärrholm and B. Schröder, Text. Res. J., 23, 207 (1953).

7. J. C. Guthrie, D. H. Morton, and P. H. Oliver, J. Text. Inst., 45, T912 (1954).

8. S. Onogi and K. Ui, J. Colloid Sci., 11, 214 (1956).

9. E. Merz, L. Nielsen, and R. Buchdahl, $J$. Polym. Sci., 4, 605 (1949).

10. A. W. Nolle, ibid., 5, 1 (1950).

11. D. A. Oliver, Phil. Mag., 14, 318 (1932).

12. M. Horio and S. Onogi, J. Appl. Phys., 22, 971 (1951).

13. S. Onogi, S. Ando, and T. Yamamoto, Kogyo Kagaku Zasshi (J. Chem. Soc. Japan, Chem. Ind. Sect.), 57, 251, 253 (1954).

14. K. Sezawa, Bull. Earthquake Researches Inst., Japan, 3, 50 (1927).

15. K. Suyehiro, Proc. Imperial Academy (Tokyo), 4, 263 (1928).

16. M. Horio and S. Onogi, J. Appl. Phys., 22, 977 (1951). 